\title{
Development of the Distance Earth-Moon
}

\author{
Dr. Andreas Gimsa \\ Stirling Technologie Institute gemeinnützige GmbH D-14478 Potsdam, Germany
}

\begin{abstract}
The expansion of the universe has a much larger dimension than previously thought. Because of entropy constancy, all masses must fall and all distances must increase. This is investigated using the example of the development of the distance Earth-Moon. The influence of tidal friction on the increase in distance is investigated and quantified. It was found that tidal friction exerts less influence than is currently assumed. If the influence of expansion and tidal friction is taken into account, a very good agreement between the calculations made here and the measured distance is achieved.
\end{abstract}

Keywords: Distance Earth-Moon, lunar laser ranging, mass decay, entropy of the universe, tidal friction, gravitational constant

\section{Introduction}

The universe is a enclosed system. There can be no transport of energy and matter across the system boundaries. The expansion of space takes place isentropic, i.e. with constant entropy. The number of effects in the universe must remain constant. The following formula applies to the entropy of the universe [01]:

$$
\mathrm{S}_{\text {uni }}=\frac{\mathrm{k}}{\hbar} \mathrm{m}_{\text {uni }} \mathrm{t}_{\text {uni }} \mathrm{C}^{2}=\frac{\mathrm{k}}{\hbar} \mathrm{N} \hbar=\mathrm{Nk}
$$

$\mathrm{N}$ has the value of $6.510^{121}$ effects. This can be interpreted as potential information, or as the number of microstates of the universe. So the mass $m_{u n i}$ of the universe must fall with its age $t_{u n i}$. With it the masses of all celestial bodies with any mass $\mathrm{m}_{\mathrm{x}}$ fall. The following applies: $\mathrm{m}_{\mathrm{x}} \mathrm{t}_{\mathrm{uni}}=$ const.

For the development of the masses of the Earth and Moon $\mathrm{m}_{1}$ and $\mathrm{m}_{2}$ over time, the following relationship then applies:

$$
\mathrm{m}_{1 \mathrm{~b}}=\frac{\mathrm{m}_{1 \mathrm{a}}}{1+\frac{\Delta \mathrm{t}}{\mathrm{t}_{\text {uni }} \mathrm{a}}} \quad \mathrm{m}_{2 \mathrm{~b}}=\frac{\mathrm{m}_{2 \mathrm{a}}}{1+\frac{\Delta \mathrm{t}}{\mathrm{t}_{\text {uni } \mathrm{a}}}}
$$

The assumption of a universal mass decay is already published in source [01]. The index a designates the respective calculation value today and the index $b$ the respective calculation value after one year $(\Delta t=1 \mathrm{a})$.

Table 1: Input values of the calculation

\begin{tabular}{|l|l|l|}
\hline Boltzmann constant & $k$ & $1.38064900 \mathrm{E}-23 \mathrm{~J} / \mathrm{K}$ \\
\hline Planck's constant & $\hbar$ & $1.05457182 \mathrm{E}-34 \mathrm{Js}$ \\
\hline Speed of light & $c$ & $2.99792458 \mathrm{E}+08 \mathrm{~m} / \mathrm{s}$ \\
\hline Gravity value & $\gamma$ & $6.67300000 \mathrm{E}-11 \mathrm{~m}^{3} /\left(\mathrm{kg} \mathrm{s}^{2}\right)$ \\
\hline Age of the universe & $t_{\text {uni }}$ & $4.35196800 \mathrm{E}+17 \mathrm{~s}$ \\
\hline Mass of the universe & $m_{\text {uni }}$ & $1.75722218 \mathrm{E}+53 \mathrm{~kg}$ \\
\hline Earth mass & $m_{1}$ & $5.97700000 \mathrm{E}+24 \mathrm{~kg}$ \\
\hline Moon mass & $m_{2}$ & $7.35200000 \mathrm{E}+22 \mathrm{~kg}$ \\
\hline Earth radius & $r_{1}$ & $6.37100100 \mathrm{E}+06 \mathrm{~m}$ \\
\hline Centre distance Earth-Moon & $r_{a}$ & $3.84400000 \mathrm{E}+08 \mathrm{~m}$ \\
\hline Day length & $T_{1}$ & $8.64000000 \mathrm{E}+04 \mathrm{~s}$ \\
\hline
\end{tabular}




\section{The time base of all oscillating systems increases with the age of the universe}

With the age of the universe $t_{u n i}$ the time base of all periodic processes must change. Since all masses become smaller, the time base of periodic processes must increase. For example, every photon becomes less energetic, its mass equivalent and its frequency become smaller: $\mathrm{mc}^{2}=\mathrm{hf}=\mathrm{h} / \mathrm{T}$. In general the periods increase with decreasing masses:

$$
\frac{\mathrm{T}_{\mathrm{a}}}{\mathrm{t}_{\text {uni } \mathrm{a}}}=\frac{\mathrm{T}_{\mathrm{b}}}{\mathrm{t}_{\text {uni } \mathrm{b}}}=\frac{\mathrm{T}_{\mathrm{b}}}{\mathrm{t}_{\text {uni } \mathrm{a}}+\Delta \mathrm{t}} \quad \mathrm{T}_{\mathrm{b}}=\mathrm{T}_{\mathrm{a}}\left(1+\frac{\Delta \mathrm{t}}{\mathrm{t}_{\text {uni } \mathrm{a}}}\right)
$$

Atomic clocks keep their resonance frequency value constant, e.g. the caesium atomic clock the frequency of 9,192,631,770 Hz. This corresponds to one second. However, the frequency of all oscillations decreases with the mass decay, e.g. the frequency of the atomic oscillation from 9,192,631,770 Hz to 9,192,631,769.33 $\mathrm{Hz}$ after one year. The second is still assigned to the old frequency value and thus remains constant. If now for the distance measurement the running time of light is measured with this time scale, where one second is smaller than it should be, more seconds pass for the light path and a larger distance comes out. This distance is then actually as it is needed for distance measurement without time change.

If, as in nature, both the distance and all periods would increase with the age of space, the decay-related distance growth would not be measurable at all. It is therefore an advantage for distance measurement that today's atomic clocks do not take the natural time base into account correctly.

\section{The enlargement of all distances}

For the drilling radius of the hydrogen atom in the lowest energy state the relation $\mathrm{r}_{\mathrm{bohr}}=\hbar /(\alpha \mathrm{c} \mathrm{m})$ applies. This means that with the mass decay of the electron the hydrogen atom must increase.

In the context of equations (02) and (03), size growth applies to all atomic distances and other distances. They increase with mass decay:

$$
r_{b}=r_{a}\left(1+\frac{\Delta t}{t_{\text {uni a }}}\right)
$$

With (04) it can be calculated immediately to what extent the distance of the moon from the earth increases, namely by $\Delta \mathrm{r}=2.8 \mathrm{~cm} / \mathrm{a}$.

In the two-mass system the center distance between the two celestial bodies can be calculated with the common period, both masses and the gravitational value. With this formula the result from equation (04) shall be verified:

$$
r=\sqrt[3]{\frac{T^{2} \gamma\left(m_{1}+m_{2}\right)}{4 \pi^{2}}}
$$

\section{The Evolution of the gravitational value with the age of the universe}

If all masses and periods change with the age of the universe, the question arises whether the gravitational value $\gamma$ also changes with time.

Paul Dirac had already postulated a variable gravitational value. With him it should decrease with time. In order to maintain the atomic structure, the ratio of the electric force $F_{e}$ to the gravitational force $F_{g}$ between the proton $\mathrm{m}_{\mathrm{p}}$ and the electron $\mathrm{m}_{\mathrm{e}}$ must remain constant:

$$
\frac{F_{e}}{F_{g}}=\frac{\frac{e^{2}}{4 \pi \varepsilon_{0} r^{2}}}{\frac{\gamma m_{p} m_{e}}{r^{2}}}=\frac{\frac{e^{2}}{4 \pi \varepsilon_{0}}}{\gamma m_{p} m_{e}}=\text { const. }
$$

Because all masses fall, the masses of proton and electron must also fall according to equation (02). Since the elementary charge e and the electric field constant $\varepsilon_{0}$ are constant, this means that the gravitational value $\gamma$ must increase:

$$
\gamma \mathrm{m}_{\mathrm{p}} \mathrm{m}_{\mathrm{e}}=\text { const. }
$$

All masses must fall in the same proportion over time, so it is generally accepted:

$$
\gamma \mathrm{m}^{2}=\text { const. }
$$


Because of the validity of equation (02), the development of the gravitational value over time can now be calculated with (08) as follows:

$$
\gamma_{\mathrm{b}}=\gamma_{\mathrm{a}}\left(1+\frac{\Delta \mathrm{t}}{\mathrm{t}_{\text {uni a }}}\right)^{2}
$$

\section{The calculation of the distance development earth-moon}

For the increase of the gap after one year $\Delta$ r results:

$$
\Delta \mathrm{r}=\mathrm{r}_{\mathrm{b}}-\mathrm{r}_{\mathrm{a}}
$$

With equation (05) the distance increase can now be calculated:

$$
\Delta \mathrm{r}=\sqrt[3]{\frac{\mathrm{T}_{\mathrm{b}}{ }^{2} \gamma_{\mathrm{b}}\left(\mathrm{m}_{1 \mathrm{~b}}+\mathrm{m}_{2 \mathrm{~b}}\right)}{4 \pi^{2}}}-\sqrt[3]{\frac{\mathrm{T}_{\mathrm{a}}{ }^{2} \gamma_{\mathrm{a}}\left(\mathrm{m}_{1 \mathrm{a}}+\mathrm{m}_{2 \mathrm{a}}\right)}{4 \pi^{2}}}
$$

If equations (02), (03) and (09) are inserted into equation (11), the following expression results:

$$
\Delta \mathrm{r}=\sqrt[3]{\frac{\mathrm{T}_{\mathrm{a}}{ }^{2} \gamma_{\mathrm{a}}\left(\mathrm{m}_{1 \mathrm{a}}+\mathrm{m}_{2 \mathrm{a}}\right)}{4 \pi^{2}}\left(1+\frac{\Delta \mathrm{t}}{\mathrm{t}_{\text {uni }}}\right)^{3}}-\sqrt[3]{\frac{\mathrm{T}_{\mathrm{a}}{ }^{2} \gamma_{\mathrm{a}}\left(\mathrm{m}_{1 \mathrm{a}}+\mathrm{m}_{2 \mathrm{a}}\right)}{4 \pi^{2}}}
$$

The simplification leads to the following calculation formula:

$$
\Delta \mathrm{r}=\sqrt[3]{\frac{\mathrm{T}_{\mathrm{a}}{ }^{2} \gamma_{\mathrm{a}}\left(\mathrm{m}_{1 \mathrm{a}}+\mathrm{m}_{2 \mathrm{a}}\right)}{4 \pi^{2}}} \frac{\Delta \mathrm{t}}{\mathrm{t}_{\text {uni a }}}
$$

Using the known quantities from equation (13), we arrive at the size growth of the distance $\Delta \mathrm{r}=2.8 \mathrm{~cm} / \mathrm{a}$ in accordance with equation (04). Currently, a distance growth of $\Delta \mathrm{r}=3.8 \mathrm{~cm} / \mathrm{a}$ is measured with special reflectors [02]. The actual distance can deviate from this distance due to the limited measuring accuracy.

\section{The influence of tidal friction on the distance development earth-moon}

The energy released by the tides is taken from the rotational movement of the earth and the angular momentum of the earth's rotation $\mathrm{L}_{1}$ around its own axis with period $\mathrm{T}_{1}$ is fed to the orbital angular momentum of the moon $\mathrm{L}_{2}$ with period $\mathrm{T}_{2}$. Thereby the day shall be extended by $16.5 \mu \mathrm{s} / \mathrm{a}$ and the distance Earth-Moon shall be increased accordingly by $\Delta \mathrm{r}=3.8 \mathrm{~cm} / \mathrm{a}$.

The total angular momentum of the Earth-Moon system must be maintained. The angular momentum of the moon's rotation around its own axis, which is bound to the moon's orbit, is comparatively small and can be neglected in this consideration. The total angular momentum at the current time must correspond to that in one year:

$$
\mathrm{L}_{1}+\mathrm{L}_{2}=\text { const. } \quad \mathrm{L}_{1 \mathrm{a}}+\mathrm{L}_{2 \mathrm{a}}=\mathrm{L}_{1 \mathrm{~b}}+\mathrm{L}_{2 \mathrm{~b}}
$$

For the calculation of the earth's angular momentum the earth is considered as an isotropic sphere with radiusr $_{1}$. In order to take into account the different mass distribution in the moment of inertia in the shell and core, a reduced mass $\mathrm{m}_{1 \mathrm{red}}=0.83 \mathrm{~m}_{1}$ is taken as a basis: $\mathrm{L}=5.8610^{33} \mathrm{~kg} \mathrm{~m}^{2} / \mathrm{s}$ [03]. Equation (14) leads with the applied angular momentum to the following expression:

$$
\frac{2}{5} m_{1 a \text { red }} r_{1 a}{ }^{2} \frac{2 \pi}{T_{1 a}}+m_{2 a} v_{2} r_{a}=\frac{2}{5} m_{1 b \text { red }} r_{1 b} \frac{2 \pi}{T_{1 b}}+m_{2 b} v_{2} r_{b}
$$

The orbital velocity of the moon $v_{2}=\frac{2 \pi r}{T} \frac{m_{1}}{m_{1}+m_{2}}$ remains constant. The influences of (02), (03) and (04) cancel each other out. Equation (15) is changed to $r_{b}$ and the difference to $r_{a}$ is formed to compare the result with (04) and (13):

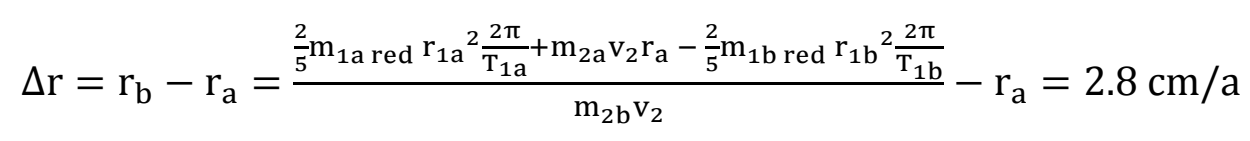


The agreement is very good, so equation (15) is then used to determine the proportion of distance increase due to tidal friction. First the growth of the rotation period $\Delta \mathrm{T}_{1}=\mathrm{T}_{1 \mathrm{~b}}-\mathrm{T}_{1 \mathrm{a}}$ of the earth is determined, which would occur after one year without tidal friction according to equation (03):

$$
\Delta \mathrm{T}_{1}=\mathrm{T}_{1 \mathrm{~b}}-\mathrm{T}_{1 \mathrm{a}}=\mathrm{T}_{1 \mathrm{a}}\left(1+\frac{\Delta \mathrm{t}}{\mathrm{t}_{\text {uni a }}}\right)-\mathrm{T}_{1 \mathrm{a}}=\mathrm{T}_{1 \mathrm{a}} \frac{\Delta \mathrm{t}}{\mathrm{t}_{\mathrm{uni}} \mathrm{a}}=6.310^{-6} \mathrm{~s} / \mathrm{a}
$$

The observed annual extension of one day is $16.5 \mu \mathrm{s}$ [04]. That means the part of the tidal friction that brakes the earth and increases the distance earth-moon is $\Delta \mathrm{T}_{1}=16.5 \mu \mathrm{s}-6.3 \mu \mathrm{s}=10.2 \mu \mathrm{s} \quad\left(\mathrm{T}_{1 \mathrm{~b}}=\mathrm{T}_{1 \mathrm{a}}+\right.$ $10.2 \mu \mathrm{s}$ ). If equation (16) is now used in such a way that the masses $\mathrm{m}_{1}, \mathrm{~m}_{2}$, the Earth radius $\mathrm{r}_{1}$ and the orbital period of the Moon $\mathrm{T}_{2}$ around the Earth remain constant, one gets the pure tidal influence on the increase in distance. It amounts to:

$$
\Delta \mathrm{r}=\mathrm{r}_{\mathrm{b}}-\mathrm{r}_{\mathrm{a}}=\frac{\frac{2}{5} \mathrm{~m}_{1 \text { red }} \mathrm{r}_{1}^{2} \frac{2 \pi}{\mathrm{T}_{1 \mathrm{a}}}+\mathrm{m}_{2 \mathrm{a}} \mathrm{v}_{2} \mathrm{r}_{\mathrm{a}}-\frac{2}{5} \mathrm{~m}_{1 \text { red }} \mathrm{r}_{1}^{2} \frac{2 \pi}{\mathrm{T}_{1 \mathrm{~b}}}}{\mathrm{~m}_{2 \mathrm{a}} \mathrm{v}_{2}}-\mathrm{r}_{\mathrm{a}}=0.9 \mathrm{~cm} / \mathrm{a}
$$

The influence of tidal friction is therefore much less than is currently assumed. Even if one adds with (18) the whole day extension of $16.5 \mu$ s to the distance increase earth-moon, one comes only to $1.5 \mathrm{~cm} / \mathrm{a}$. This is not even half of the observed value and therefore the calculation with temporally unchanging masses is doubtful.

The calculated total growth of the distance earth-moon from the equations (16) and (18) is: $\Delta \mathrm{r}=$ $(2.8+0.9) \mathrm{cm} / \mathrm{a}=3.7 \mathrm{~cm} / \mathbf{a}$. This value is very close to the measured value of $3.8 \mathrm{~cm} / \mathrm{a}$ and is plausible.

\section{Uncertainties}

The age of the universe and the influence of other celestial bodies on the two-mass system Earth-Moon, e.g. the sun, can affect the actual distance and lead to deviations from the calculations according to equations (04), (13), (16) and (18).

\section{Outlook}

Double star systems, which are very common in the universe, would have to behave in the same way. Both stars are moving away from each other. Therefore, it is conceivable that such systems have evolved from a single star. The view in cosmology that double stars collapse under the emission of gravitational waves is reversed: Due to the mass decay, both stars of a double star system move away from each other.

\section{Summary}

The calculated Earth-Moon distance growth agrees quite well with the observation. The expansion of space should cause a global mass decay and an enlargement of all distances and thus also of all scales.

If equation (04) is used to determine the increase in the radius of the earth, Pascual Jordan's result is obtained. He has described an annual growth of $\Delta \mathrm{r}_{1}=0.5 \mathrm{~mm}$ [05].

\section{References}

[1.] Andreas Gimsa, The metric of space-time, English-German, 2nd edition, Pots- dam 2020, ISBN 978-3-00-064784-0

[2.] Jürgen Müller, Why you measure the distance to the moon, Yearbook of scientific society Braunschweig, p.24, Leibnitz University of Hannover, Institute for Earth Measurement, 2018, (see also Lunar Laser Ranging)

[3.] Parameters of Earth Rotation and Global Dynamical Systems, Federal Agency for Cartography and Geodesy, Frankfurt am Main, Germany, 2020

[4.] Walter Greiner, Theoretical Physics, Classical Mechanics I, p.280 Scientific publisher Harri Deutsch GmbH, Frankfurt am Main, 2008

[5.] Pascual Jordan, The Expansion of the Earth, p.77, Vieweg-Verlag Braun- schweig, 1966 